\title{
LOCALLY FLAT, LOCALLY TAME, AND TAME EMBEDDINGS
}

\author{
BY CHARLES GREATHOUSE
}

Communicated by Deane Montgomery, May 23, 1963

1. Introduction. Brown [1] has shown that an $S^{n-1}$ embedded in a locally flat manner in $S^{n}$ is flat and hence tame in $S^{n}$. Bing [2] and Moise [3] have shown that locally tame subsets of 3-manifolds are tame. However, in the general case, it is not known whether a manifold $N$ embedded in a locally flat manner in a triangulated manifold $M$ or a polyhedron $P$ embedded in a locally tame manner in a triangulated manifold $M$ are tame in $M$. Partial solutions to both of these problems have been obtained by the author and will be stated in $\$ 3$ of this paper. I have been informed by R. H. Bing that Herman Gluck has obtained similar results.

2. Definitions and notations. Let $N^{k}$ be a combinatorial $k$-manifold. Then $\left(N^{k}\right)^{r}$ will denote the $r$ th barycentric subdivision of $N^{k}$. If $\alpha$ is a $k$-simplex of $\left(N^{k}\right)^{r}$ and $\alpha^{\prime \prime}$ is the union of all simplexes of $\left(N^{k}\right)^{r+2}$ contained in $\alpha$, then $C_{\alpha}$ will denote the closed simplicial neighborhood of $\left|\alpha^{\prime \prime}\right|$, the polyhedron of $\alpha^{\prime \prime}$, in $\left(N^{k}\right)^{r+2}$. That is $C_{\alpha}$ is the union of all closed simplexes in $\left(N^{k}\right)^{r+2}$ that meet $\left|\alpha^{\prime \prime}\right|$. Since $\alpha^{\prime \prime}$ is collapsible, $C_{\alpha}$ is a combinatorial $k$-ball [4].

The statement that $f$ is a locally flat embedding of a $k$-manifold $N^{k}$ in an $n$-manifold $N^{n}$, means that each point of $f\left(N^{k}\right)$ has a neighborhood $U$ in $N^{n}$ such that the pair $\left(U, U \cap f\left(N^{k}\right)\right)$ is homeomorphic to the pair $\left(R^{n}, R^{k}\right)$.

Two definitions of locally tame will now be given.

Definition 1. Let $N$ be a manifold topologically embedded in a triangulated manifold $M . N$ is locally tame if for each point $p$ of $N$, there exists a neighborhood $U$ of $p$ in $M$ and a homeomorphism $h$ of $\bar{U}$ into $M$, such that $h[\mathrm{Cl}(U \cap N)]$ is a polyhedron in $M$.

Definition 2. Let $P$ be a polyhedron topologically embedded in a triangulated manifold $M . P$ is locally tame if for each point $p$ of $P$, there exists a neighborhood $U$ of $p$ in $M$ and a homeomorphism $h$ of $\bar{U}$ into $M$, such that $h \mid \mathrm{Cl}(U \cap P)$ is piecewise linear with respect to a fixed triangulation $T$ of $P$.

Let $K$ be a complex topologically embedded by $f$ in a triangulated $n$-manifold $N^{n}$ and let $\epsilon>0$. Suppose there exists an $\epsilon$-homeomorphism $h$ of $N^{n}$ onto itself such that if $U_{\epsilon}(f(K))$ denotes the set of points in $N^{n}$ whose distance from $f(K)$ is less than $\epsilon$, then 
(i) $h \mid N^{n}-U_{\mathrm{e}}(f(K))=1$,

(ii) $h f: K \rightarrow N^{n}$ is a piecewise linear embedding.

Then $f(K)$ will be said to be $\epsilon$-tame in $N^{n}$.

3. Statement of results.

TheOREM 1. Let $f$ be a locally flat embedding of a closed combinatorial $k$-manifold $N^{k}$ in a closed combinatorial $n$-manifold $N^{n}, 2 k+2 \leqq n$ and $\epsilon>0$. Then $f\left(N^{k}\right)$ is $\epsilon$-tame in $N^{n}$.

TheOREM 2. Let $f_{1}$ and $f_{2}$ be locally flat (locally tame) embeddings of a closed combinatorial $k$-manifold $N^{k}$ (finite k-polyhedron $P^{k}$ ) in $S^{n}$ and $2 k+2 \leqq n$. Then there exists a homeomorphism $h$ of $S^{n}$ onto itself such that $h f_{1}=f_{2}$.

THEOREM 3. Let $f$ be a locally flat embedding of a $k$-manifold $N^{k}$ in a combinatorial $n$-manifold $N^{n}$ and $2 k+2 \leqq n$. Then $f\left(N^{k}\right)$ is locally tame (Definition 1).

THEOREM 4. Let $f$ be a locally tame (Definition 2) embedding of a possibly infinite $k$-polyhedron $P^{k}$ as a closed subset of the interior of a combinatorial $n$-manifold $N^{n}, 2 k+2 \leqq n$ and $\epsilon>0$. Then $f\left(P^{k}\right)$ is $\epsilon$-tame in $N^{n}$.

\section{Reference theorems.}

Homma's THEOREM [5]. Let $M^{n}, \hat{M}^{n}$ and $\hat{P}^{k}$ be two finite combinatorial $n$-manifolds and a finite polyhedron such that $\hat{M}^{n}$ is topologically embedded in $M^{n}, \hat{P}^{k}$ is piecewise linearly embedded in $\operatorname{Int}\left(\hat{M}^{n}\right)$ and $2 k+2 \leqq n$. Then for $\epsilon>0, \hat{P}^{k}$ is $\epsilon$-tame in $M^{n}$.

Gluck's MODIfication of Homma's theOREm [6]. Let the following be given:

(i) $M^{n}$, a possibly noncompact combinatorial $n$-manifold;

(ii) $\hat{M}^{n}$, a possibly noncompact combinatorial $n$-manifold, topologically embedded in $M^{n}$;

(iii) $\hat{P}^{k}$, a possibly infinite polyhedron, piecewise linearly embedded as a closed subset of $\operatorname{Int}\left(\hat{M}^{n}\right)$;

(iv) $\hat{L}$, a subpolyhedron of $\hat{P}^{k}$ such that $\mathrm{Cl}\left(\hat{P}^{k}-\hat{L}\right)$ is a finite polyhedron, and such that $\hat{L}$ is piecewise linearly embedded in $M^{n}$ as well as in $\hat{M}^{n}$.

If $2 k+2 \leqq n$, then for any $\epsilon>0$, there is an $\epsilon$-homeomorphism $F$ of $M^{n}$ onto $M^{n}$ such that under $F, \hat{P}^{k}-\hat{L}$ is $\epsilon$-tame in $M^{n}$ and $F \mid \hat{L}=1$.

\section{Partial proofs of results.}

LEMмA 1. Suppose the following are given: 
(i) The hypotheses of Theorem 1 are satisfied.

(ii) $\left\{\left(U_{i}, U_{i} \cap f\left(N^{k}\right)\right), i=1, \cdots, q\right\}$ is a finite open cover of $f\left(N^{k}\right)$ obtained by applying the definition of locally flat.

(iii) $\epsilon>0$.

Then there exists an integer $r$ such that if $\alpha$ is a $k$-simplex of $\left(N^{k}\right)^{r}$ and if $C_{f(\alpha)}=f\left(C_{\alpha}\right)$,

(a) $f(\alpha) \subset C_{f(\alpha)} \subset U_{j} \cap f\left(N^{k}\right)$ for some $j$.

(b) $C_{f(\alpha)}$ is $\epsilon$-tame in $N^{n}$.

Conclusion (a) is obvious since every open cover of a compact metric space has a Lebesgue number and the limit of the mesh of $f\left(N^{k}\right)^{i}$ as $i$ approaches infinity is zero.

Let $r$ and $j$ be integers such that conclusion (a) is true. Let $h_{j}$ be the homeomorphism of $\left(U_{j}, U_{j} \cap f\left(N^{k}\right)\right)$ onto $\left(R^{n}, R^{k}\right)$. Since $\dot{C}_{\alpha}$ is a bicollared [1] $k-1$ sphere in $N^{k}, h_{j}\left(f\left(\dot{C}_{\alpha}\right)\right)$ is a bicollared $k-1$ sphere in $R^{k}$. Hence $h_{j}\left(C_{f(\alpha)}\right)$ is a tame $k$-cell in $R^{k}$ and therefore $U_{j}$ can be triangulated as a combinatorial $n$-manifold in such a way that $f: C_{\alpha} \rightarrow U_{j}$ is a piecewise linear embedding.

We now apply Homma's theorem. Let $M^{n}=N^{n}, \hat{M}^{n}=$ a closed regular neighborhood of $C_{f(\alpha)}$ in $U_{j}$ and $\hat{P}^{k}=C_{f(\alpha)}$. Homma's theorem asserts that $C_{f(\alpha)}$ is $\epsilon$-tame in $N^{n}$.

Proof of TheOREM 1. Let $r$ be an integer such that if $\alpha$ is a $k$ simplex of $\left(N^{k}\right)^{r}$, Lemma 1 is valid. Let $A_{i}$ denote the proposition that if $K_{i}$ is a connected homogeneous $k$-subcomplex of $\left(N^{k}\right)^{r}$ containing $i$ $k$-simplexes, then $f\left(K_{i}\right)$ is $\epsilon$-tame in $N^{n}$ for each $\epsilon>0$. It suffices to show that $A_{i}$ is true for each positive integer $i$.

$A_{1}$ is true by Lemma 1 . Suppose $A_{i}$ is true for $1 \leqq i \leqq j$. Let $K_{j+1}$ be a connected homogeneous $k$-subcomplex of $\left(N^{k}\right)^{r}$ containing $j+1$ $k$-simplexes. Then $K_{j+1}=K_{j} \cup_{\alpha}$, where $K_{j}$ is a connected homogeneous $k$-subcomplex of $\left(N^{k}\right)^{r}$ containing $j k$-simplexes and $\alpha$ is a $k$-simplex of $\left(N^{k}\right)^{r}$. Let $\epsilon>0$ and $\epsilon^{\prime}=\epsilon / 2$, then by assumption, $f\left(K_{j}\right)$ is $\epsilon^{\prime}$-tame in $N^{n}$ and by Lemma $1, C_{f(\alpha)}$ is $\epsilon^{\prime}$-tame in $N^{n}$.

Let $h_{k}$ and $h_{\alpha}$ be the $\epsilon^{\prime}$-homeomorphisms for $f\left(K_{j}\right)$ and $C_{f(\alpha)}$ respectively such that they are $\epsilon^{\prime}$-tame in $N^{n}$. Let $U_{\alpha}$ be an open ball neighborhood of $h_{\alpha}\left(C_{f(\alpha)}\right)$ in $N^{n}$, and $W_{\alpha}=h_{\alpha}{ }^{-1}\left(U_{\alpha}\right)$.

We will complete the proof of $A_{j+1}$ by applying Gluck's modification of Homma's theorem. Let $M^{n}=h_{k}\left(w_{\alpha}\right)$ triangulated as an open subset of $N^{n}, \hat{M}^{n}=h_{k}\left(W_{\alpha}\right)$ triangulated as a combinatorial $n$-manifold such that $h_{k} f: C_{\alpha} \rightarrow h_{k}\left(W_{\alpha}\right)$ is a piecewise linear embedding. Take $\hat{P}^{k}=h_{k}\left[C_{f(\alpha)} \cap f\left(K_{j}\right)\right] \cup h_{k}(f(\alpha))$ and $\hat{L}=h_{k}\left[C_{f(\alpha)} \cap f\left(K_{j}\right)\right]$. By choice of $h_{k}, \hat{L}$ is piecewise linearly embedded in both $M^{n}$ and $\hat{M}^{n}$. Let $\epsilon^{\prime \prime}$ be picked such that $0<\epsilon^{\prime \prime}<\epsilon^{\prime}$ and such that $\left[U_{\epsilon^{\prime \prime}}\left(h_{k}(f(\alpha))\right)\right] \cap h_{k}\left(f\left(K_{j}\right)\right) \subset \hat{L}$ 
and $\mathrm{Cl}\left[U_{\mathrm{\epsilon}^{\prime \prime}}\left(h_{k}(f(\alpha))\right)\right] \subset h_{k}\left(W_{\alpha}\right)$. The hypotheses of Gluck's theorem are satisfied, hence there exists an $\epsilon^{\prime \prime}$-homeomorphism $g$ of $M^{n}$ onto itself such that $\hat{P}^{k}-\hat{L}$ is $\epsilon^{\prime \prime}$-tame in $M^{n}$ under $g$ and $g \mid \hat{L}=1$. $g$, which is the identity on $h_{k}\left[f\left(K_{j}\right) \cap W_{\alpha}\right]$ and near the boundary of $h_{k}\left(W_{\alpha}\right)$, may be extended via the identity to an $\epsilon^{\prime \prime}$-homeomorphism $\bar{g}$ of $N^{n}$ onto itself.

Then $F=\bar{g} h_{k}$ is an $\epsilon$-homeomorphism of $N^{n}$ onto itself, such that under $F, f\left(K_{j+1}\right)$ is $\epsilon$-tame in $N^{n}$. Thus $A_{j+1}$ is true and by induction the theorem is proved.

Theorems 1 and 4 reduce the proof of Theorem 2 to the piecewise linear case which has already been handled in [7].

The proof of Theorem 3 is an easy application of Homma's theorem. The following lemma also follows from Homma's theorem.

LemMa 2. Suppose the following are given:

(i) The hypotheses of Theorem 4 are satisfied except $P^{k}$ is finite.

(ii) $\left\{\left(U_{\lambda}, U_{\lambda} \cap f\left(P^{k}\right), \lambda=1, \cdots, q\right\}\right.$ is a finite open cover of $f\left(P^{k}\right)$ obtained by applying Definition 2 of locally tame.

(iii) $\epsilon>0$.

Then there exists a triangulation of $f\left(P^{k}\right)$ such that the closed simplicial neighborhood of any simplex in this triangulation of $f\left(P^{k}\right)$ is contained in $U_{j} \cap f\left(P^{k}\right)$ for some $j$ and is $\epsilon$-tame in $N^{n}$.

Lemma 2, together with Gluck's modification of Homma's theorem are sufficient to prove Theorem 4.

Actually, Lemma 1 shows that locally flat closed combinatorial manifolds with the correct codimension are locally tame according to Definition 2. This, together with Theorem 4, would yield Theorem 1 as a corollary.

\section{REFERENCES}

1. M. Brown, Locally flat imbeddings of topological manifolds, Ann. of Math. (2) 75 (1962), 331-341.

2. R. H. Bing, Locally tame sets are tame, Ann. of Math. (2) 59 (1954), 145-158.

3. E. E. Moise, Affine structures in 3-manifolds. VIII, Ann. of Math. (2) 59 (1954), 159-170.

4. J. H. C. Whitehead, Simplicial spaces, nuclei and m-groups, Proc. London Math. Soc. (2) 45 (1939), 243-327.

5. T. Homma, On the imbedding of polyhedra in manifolds, Yokohama Math. J. 10 (1962), 5-10.

6. Herman Gluck, Unknotting $S^{1}$ in $S^{4}$, Bull. Amer. Math. Soc. 69 (1963), 91-94.

7. V. K. A. M. Gugenheim, Piecewise linear isotopy and embedding of elements and spheres. I, Proc. London Math. Soc. (3) 3 (1953), 29-53.

Florida State University 